Note

\title{
FRACTIONAL ASPECTS OF THE ERDŐS-FABER-LOVÁSZ CONJECTURE
}

\author{
John Bosica and Claude Tardif \\ Department of Mathematics and Computer Science \\ Royal Military College of Canada \\ PO Box 17000 Stn Forces, Kingston, ON Canada, K7K $7 B 4$ \\ e-mail: John.Bosica@rmc.ca \\ Claude.Tardif@rmc.ca
}

\begin{abstract}
The Erdős-Faber-Lovász conjecture is the statement that every graph that is the union of $n$ cliques of size $n$ intersecting pairwise in at most one vertex has chromatic number $n$. Kahn and Seymour proved a fractional version of this conjecture, where the chromatic number is replaced by the fractional chromatic number. In this note we investigate similar fractional relaxations of the Erdős-Faber-Lovász conjecture, involving variations of the fractional chromatic number. We exhibit some relaxations that can be proved in the spirit of the Kahn-Seymour result, and others that are equivalent to the original conjecture.
\end{abstract}

Keywords: Erdős-Faber-Lovász Conjecture, fractional chromatic number.

2010 Mathematics Subject Classification: 05C15, 05C72.

\section{REFERENCES}

[1] N.G. de Bruijn and P. Erdős, On a combinatorial problem, Nederl. Akad. Wetensch. Indag. Math 10 (1948) 421-423.

[2] P. Erdős, R.C. Mullin, V.T. Sós and D.R. Stinson, Finite linear spaces and projective planes, Discrete Math. 47 (1983) 49-62.

doi:10.1016/0012-365X(83)90071-7

[3] J. Kahn, Coloring nearly-disjoint hypergraphs with $n+o(n)$ colors, J. Combin. Theory (A) 59 (1992) 31-39.

doi:10.1016/0097-3165(92)90096-D

[4] J. Kahn and P.D. Seymour, A fractional version of the Erdös-Faber-Lovász conjecture, Combinatorica 12 (1992) 155-160.

doi:10.1007/BF01204719 
[5] E.R. Scheinerman and D.H. Ullman, Fractional Graph Theory (Wiley-Interscience Series in Discrete Mathematics and Optimization, John Wiley \& Sons, New York, 1997).

Received 22 May 2013

Revised 28 November 2013

Accepted 20 February 2014 